\title{
Acceptance, choice and empowerment (ACE) for pre-dialysis patients: a peer educator-based community project that aims to improve choice and quality of life for patients with kidney failure
}

\author{
Authors: Neerja Jain, ${ }^{A}$ Jyoti Baharani, ${ }^{B}$ Annette Dodds ${ }^{B}$ and Angela Lumsdon ${ }^{A}$
}

\section{Aims}

To improve patients' acceptance of their kidney failure diagnosis and to empower them regarding their dialysis treatment choices.

\section{Methods}

> Kidney Research UK has led a pilot project using its evidencebased multi-award-winning peer educator (PE) model, involving trained and accredited volunteers who have a natural empathy with the patient group through language, culture, religion and healthcare experience.

> Ten PE volunteers were recruited with nine remaining engaged during the 17-month pilot project, all of them with personal experience of renal replacement therapy.

> In partnership with patients and clinical staff (a consultant and two-three senior nurses were involved), they worked collaboratively to facilitate acceptance and decision making.

The project had a four-pronged approach:

> Visiting patients at home with the kidney nurse specialist

$>$ Meeting patients in clinic

> Phoning patients (if requested) post visit / clinic

> Organising Asian-specific patient information days (these were later broadened to include all ethnic groups).

\section{Results}

> Fourteen home visits were undertaken. One patient said: 'I was very grateful that the nurse and PE took time to provide me with information. I was scared at first but was really happy that the PE spoke to me in my own language, and explained the dialysis to me, as she has been through it.'

> Five patient information days were attended by 50 patients and 44 family members. In the past, the number of Asian patients attending such events had been poor, averaging just one per event; the bespoke events attracted almost 10 times as many

Authors: ${ }^{A}$ Kidney Research UK, Peterborough; ${ }^{B}$ on behalf of ACE project team, Glaxo Renal Unit, Heart of England NHS Foundation Trust, Birmingham
Asian patients. One said: 'It gave me a helpful insight into the reality of dialysis.' Feedback scores have been overwhelmingly positive and all patients said the project had helped them make a decision about their dialysis treatment.

$>44$ patients were seen in clinic: One patient, who found speaking with a PE very cathartic, cried but said it helped him to finally accept his diagnosis.

> One key senior staff member described their change of attitude from initially feeling negative, cynical and anxious about the project (based on previous experience of peer support) to subsequently feeling very passionate. This staff member felt it was essential to continue this service improvement!

$>$ All of the volunteer PEs expressed a commitment to continue to volunteer despite the formal end of the pilot project.

\section{Conclusion}

This innovative pilot project utilised the peer educator approach in a novel way. In a very short space of time, this pilot proved to be impactful and had very positive 'buy-in' from patients, staff and the PE volunteers. We believe some of the success can be attributed to the ethos of involving and empowering patients, working in collaboration with renal healthcare professionals for the ultimate benefit of new renal patients. The project team are highly committed and passionate about sustaining this work and helping spread to other units that would benefit from this initiative.

The project has achieved its aims of pilot testing a method to help with patient acceptance, choice and empowerment (ACE).

The knowledge gained from this pilot shows that it is feasible to recruit, train and utilise the unique skills of patients as volunteers to support other (newly diagnosed) patients to accept their chronic, life-changing condition, and make informed choices about their treatment while empowering them.

We have the proof of principle that PEs can meet the needs of renal units, working within the NHS trust volunteer/governance framework. This initiative is low-cost, demonstrating new ways of working, empowering patients and utilising non-clinical workforce.

\section{Conflict of interest statement}

No conflict of interests stated from any of the authors. 